September 3, 2021

\title{
Modification of magicity towards the dripline and its impact on electron-capture rates for stellar core-collapse
}

\author{
Ad. R. Raduta, ${ }^{1}$ F. Gulminelli, ${ }^{2}$ and M. Oertel ${ }^{3}$ \\ ${ }^{1}$ IFIN-HH, Bucharest-Magurele, POB-MG6, Romania \\ ${ }^{2}$ CNRS/ENSICAEN/LPC/Université de Caen Basse Normandy, UMR6534, F-14050 Caen cédex, France \\ ${ }^{3}$ LUTH, CNRS, Observatoire de Paris, Université Paris Diderot, 5 place Jules Janssen, 92195 Meudon, France
}

\begin{abstract}
The importance of microphysical inputs from laboratory nuclear experiments and theoretical nuclear structure calculations in the understanding of the core collapse dynamics, and the subsequent supernova explosion, is largely recognized in the recent literature. In this work, we analyze the impact of the masses of very neutron rich nuclei on the matter composition during collapse, and the corresponding electron capture rate. To this aim, we introduce an empirical modification of the popular Duflo-Zuker mass model to account for possible shell quenching far from stability, and study the effect of the quenching on the average electron capture rate. We show that the preeminence of the $N=50$ and $N=82$ closed shells in the collapse dynamics is considerably decreased if the shell gaps are reduced in the region of ${ }^{78} \mathrm{Ni}$ and beyond. As a consequence, local modifications of the overall electron capture rate up to $30 \%$ can be expected, with integrated values strongly dependent on the stiffness of magicity quenching and progenitor mass and potential important consequences on the entropy generation, the neutrino emissivity, and the mass of the core at bounce. Our work underlines the importance of new experimental measurements in this region of the nuclear chart, the most crucial information being the nuclear mass and the Gamow-Teller strength. Reliable microscopic calculations of the associated elementary rate, in a wide range of temperatures and electron densities, optimized on these new empirical information, will be additionally needed to get quantitative predictions of the collapse dynamics.
\end{abstract}

PACS numbers: $26.50 .+\mathrm{x}, 23.40 .-\mathrm{s}, 97.60 . \mathrm{Bw}$

\section{INTRODUCTION}

It is nowadays well recognized that reliable nuclear physics inputs are essential for realistic simulations of many astrophysical phenomena. Depending on the particular time scales and the resulting equilibrium conditions, this can be an equation of state (EoS) and individual (nuclear) reactions, respectively. Due to the relatively long timescale of reactions mediated by the weak interaction, the latter play an important role in many sites, for instance the late stages of massive star evolution [1-3], thermonuclear [4,5] and core-collapse supernovae [6-8], nucleosynthesis and energy generation in X-ray bursts and other rp-process sites [9], the accreting neutron star crust [10, 11], and neutron star mergers [12, 13].

Within this paper, we will concentrate on core-collapse supernovae (CCSNe). Except for very low-density matter encountered in the outer layers, time scales are such that strong and electromagnetic interactions are in equilibrium. Therefore, at any time, the composition of matter can be calculated as a function of the local temperature $(T)$, baryon number density $\left(n_{B}\right)$ and proton fraction $\left(Y_{p}\right)$ assuming Nuclear Statistical Equilibrium (NSE) (see e.g. [8]). On the contrary, weak interactions can in general not be considered in equilibrium and individual reactions rates are crucial to determine the local proton fraction. In particular, electron capture determines $Y_{p}$ in the first stages of the collapse, the associated size of the homologous core, and has thus an impact on the consequent explosion dynamics [6, 8, 14-18].

For these simulations, the time and space dependent electron capture rates are obtained for a given $\left(n_{B}, T, Y_{p}\right)$ by folding the NSE nuclear distribution with the capture rates on in- dividual nuclei. The microphysics uncertainties on the rates thus originate both from the uncertainties on the NSE distribution, and on those associated with the individual rates.

A very complete study on the sensitivity of core collapse dynamics to variations of electron capture rates on mediumheavy nuclei has recently been performed [18]. The authors thereby concentrate on the latter aspect, modifying the rates on individual nuclei according to present uncertainties, using a comprehensive set of progenitors and EoS. It was shown that important variations in the mass of the inner core at bounce and in the peak neutrino luminosity have to be expected. The variations induced by the modified electron capture rates are five times larger than those induced by the uncertainty on the progenitor model, showing the importance of an increased reliability of nuclear physics inputs. In this same work it was clearly shown that the results are most sensitive to the region around the $N=50$ shell closure for very neutron rich nuclei $(74 \leq A \leq 84)$.

In this work we address the complementary aspect of uncertainties associated with the nuclear distribution. Specifically, nuclear magicity, as incorporated in mass models within currently available EoS, is known to be deeply modified in very neutron rich nuclei [19] and the pronounced shell closures at $N=50$ and $N=82$ are expected to be quenched far from stability. We study here the impact of such a possible quenching on the matter composition during collapse, and the associated modification of the electron capture rate. We show that modifications of the electron capture rate of up to $30 \%$ are possible in the case of strong shell quenching.

Unlike Ref. [18], in this exploratory calculation we do not aim at computing the complete time evolution of $Y_{p}$, nor the associated modification of the inner core mass and the neu- 
trino luminosity. Such a complete simulation would require a consistent model for the mass and electron capture rates as determined by Fermi and Gamow-Teller transition strengths for the relevant nuclei. This is beyond the scope of the present paper. However, from general arguments and the very detailed results of Ref. [18], we expect that an increased capture rate leads to enhanced neutrino cooling, an accelerated collapse, and a higher inner core mass at bounce. The main message of the present work is to stress the need of structure information and mass measurements, particularly around the ${ }^{78} \mathrm{Ni}$ region. From the theory viewpoint, it appears very important to have microscopic and consistent calculations of both masses and weak rates.

The paper is organized as follows. In Section $[$ the different microphysical inputs entering the electron capture rate calculations are presented, namely the individual rates and the equation of state. We show that for the latter, the mass model represents the key ingredient. The influence of the mass model on the capture rates during core collapse is discussed in Section IIII. To that end, we consider two representative collapse trajectories with typical thermodynamic conditions. An effective parametrization simulating the possible shell quenching far from stability of the two relevant shell closures $N=50$ and $N=82$ is introduced, and the associated modification of the global rate is discussed. Section IV contains summary and conclusions. Throughout the paper we will use units such that $k_{B}=1$.

\section{INGREDIENTS OF THE RATE CALCULATION}

As mentioned above, for given thermodynamic condition, i.e. given values of $\left(n_{B}, T, Y_{p}\right)$, the total electron capture rate is obtained by folding individual rates with the nuclear distribution. The latter is obtained by NSE calculations which depend in turn on a number of inputs and in particular on the masses of different nuclei, thus on the mass model employed. In this section we detail the different ingredients employed in this work. We will start with the individual rates in Sec IIA. The NSE model will be discussed in Sec. IIB Sec. IIC will be devoted to the mass model and special attention will be paid to the possible quenching of magicity in very neutron rich nuclei.

\section{A. Individual rates}

Concerning the individual rates, tabulated values are available from large scale shell model calculations in the $s d$-shell [20] (OHMTS), and $f p$-shell [21, 22] (LMP). Since those calculations are still nowadays numerically very demanding, for heavier nuclei, the shell model rates are complemented with shell model Monte Carlo and RPA calculations [23] or an empirical approach [24] (PF). In Ref. [25] shell model rates on additional nuclei have been calculated, however, they are unfortunately not available as individual rates. These extended calculations still comprise a limited range of elements, masses, temperature and electron densities $\left(n_{e}=n_{p}\right)$ and are not sufficient to cover completely the typical conditions in the most central part of the core collapse, nor the typical nuclei encountered in those situations.

We have therefore decided to use here the analytical equation proposed by Langanke et al. (L03) in Ref. [7]. It is based on a parametrization of the electron-capture rate as a function of the ground state to ground state $Q$-value and writes,

$$
\lambda_{E C}=\frac{\ln 2 \cdot B}{K}\left(\frac{T}{m_{e} c^{2}}\right)^{5}\left[F_{4}(\eta)-2 \chi F_{3}(\eta)+\chi^{2} F_{2}(\eta)\right],
$$

with $K=6146 \mathrm{~s}, \chi=(Q-\Delta E) / T, \eta=\chi+\mu_{e} / T$. $T$ represents the temperature, $m_{e}$ and $\mu_{e}$ stand for electron rest mass and, respectively, chemical potential. $F_{i}(\eta)$ denotes the relativistic Fermi integral, $F_{i}(\eta)=\int_{0}^{\infty} d x x^{k} /(1+\exp (x-\eta))$. $B$ stands for a typical (Gamow-Teller plus forbidden) matrix element and $\Delta E=E_{f}-E_{i}$ represents the energy difference between the parent state $E_{i}$ and the daughter state $E_{f}$. Their values, obtained by fitting microscopic data, are $B=4.6 \mathrm{MeV}$ and $\Delta E=2.5 \mathrm{MeV}$. For electron capture rates on protons we use the results of Ref. [26].

The first approximation of weak interaction rates in terms lepton chemical potential and $Q$-value was proposed by Fuller, Fowler and Newman in the pioneering Ref. [27] in the form $\lambda_{E C}=\ln 2 I_{e} /\langle f t\rangle_{e}$, where $I_{e}$ is the space factor. Nuclear structure effects were there approximately accounted for via the effective $\log (\mathrm{ft})$-value. For EC-rates three values have been proposed based on the neutron and proton numbers of the parent nucleus (upon which nuclei are classified in "blocked" $(N \geq 40$ or $Z \leq 20)$ and "unblocked" $(N<40$ and $Z>20)$ ) and the relation between electron chemical potential and $Q$-value. For "unblocked" nuclei EC was supposed to occur mainly through the Gamow-Teller resonances which are roughly at an excitation of $3 \mathrm{MeV}$ above the daughter ground state.

The validity of the analytic equation L03 has been recurrently addressed in the literature (see e.g. [7, 18]) and proved to offer a fair approximation under thermodynamical conditions relevant for core-collapse. Scatter of microscopic rates around the $Q$-dependence at low values of temperature and electron density were interpreted in Ref. [7] as indicating that several parent and daughter states with different transition strengths contribute to the process. On the contrary, better agreement at higher values of temperature and electron density was attributed to a value of the electron chemical potential high enough to make EC-rate independent of the strength distribution. Fig. 11addresses the same issue by confronting the predictions of eq. (1), plotted as a function of reaction heat $Q$, with those obtained by linear interpolation of more microscopic and sophisticated weak interaction rate tables OHMTS [20] (panels (a) and (d)), LMP [21, 22] (panels (b) and (e)) and PF [24] (panels (c) and (f)). Two representative thermodynamic conditions taken from the core-collapse trajectory of a $25 M_{\odot}$ star in Ref. [25] are considered (see figure caption). As already evidenced by other authors, Fig. 1 shows a correct overall behavior of $\lambda_{E C}(Q)$ and, in what concerns the individual rates, important deviations. Quite remarkably, eq. (1) offers a fair approximation also outside the mass range for which it was designed. 

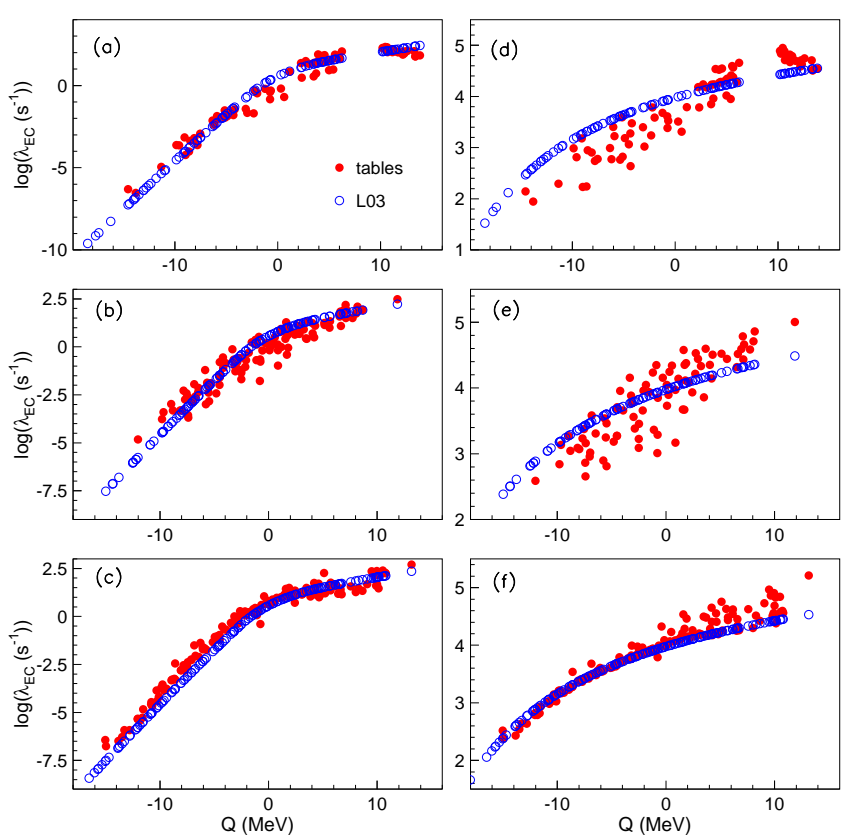

FIG. 1: (Color online) EC rates: comparison between L03 (open blue circles) and OHMTS [20] $(17 \leq A \leq 39)(\mathrm{a}, \mathrm{d})$, LMP [21, 22] $(45 \leq A \leq 65)(\mathrm{b}, \mathrm{e})$ and $\mathrm{PF}[24](65 \leq A \leq 80)$ (c,f) tables (solid red circles) for $T=0.68 \mathrm{MeV}$, $n_{B}=1.32 \cdot 10^{-6} \mathrm{fm}^{-3}, Y_{e}=0.447(\mathrm{a}, \mathrm{b}, \mathrm{c})$ and $T=1.30 \mathrm{MeV}$, $n_{B}=1.12 \cdot 10^{-4} \mathrm{fm}^{-3}, Y_{e}=0.361(\mathrm{~d}, \mathrm{e}, \mathrm{f})$.

Whenever exist the deviations underline the well known fact that, in order to accurately describe astrophysical processes, it will be very important to have fully microscopic calculations covering the whole mass, charge, temperature and electron fraction domain. It was recently demonstrated in Ref. [18] that a global arbitrary re-scaling of a factor 2,3 or 10 of the unknown rates leads to important modifications in the dynamics of the collapse. Given the purpose of this paper, which is to analyze the influence of the mass extrapolations in the still experimentally unknown region of very neutron rich nuclei, we have not tried to play with these rates, and we consider that for our purpose the L03 equations are sufficiently accurate to provide a reference calculation.

\section{B. Extended NSE model}

The model is based on a statistical distribution of compressible nuclear clusters composed by $A$ nucleons ( $N$ neutrons and $Z$ protons) immersed in a homogeneous background of selfinteracting nucleons and electrons. The details of the model are explained elsewhere [28, 29]; here we only recall the main physical ingredients which are important for the present study.

The different thermodynamic quantities in the baryonic sector are decomposed into the sum of a term pertaining to the nucleon gas, and a term given by the contributions of the distribution of nuclei. Let us start with the gas contribu- tion. In absence of clusters, it would simply be given by the free energy density of homogeneous nuclear matter at density $n_{g}=n_{g n}+n_{g p}$, asymmetry $\delta_{g}=\left(n_{g n}-n_{g p}\right) / n_{g}$ and temperature $T$. In the non-relativistic mean field approximation it reads $(q=n, p)$ :

$$
\begin{aligned}
f_{H M}\left(n_{g}, \delta_{g}\right) & =\sum_{q} g_{q} \int_{0}^{\infty} \frac{d p p^{2}}{2 \pi^{2} \hbar^{3}} n_{q}^{T} \frac{p^{2}}{2 m_{q}^{*}}+ \\
& +\mathscr{E}_{p o t}-T s_{H M},
\end{aligned}
$$

with the entropy density given by :

$$
\begin{aligned}
s_{H M}\left(n_{g}, \delta_{g}\right) & =-\sum_{q} g_{q} \int_{0}^{\infty} \frac{d p}{2 \pi^{2} \hbar^{3}} p^{2}\left[n_{q}^{T} \ln n_{q}^{T}+\right. \\
& \left.+\left(1-n_{q}^{T}\right) \ln \left(1-n_{q}^{T}\right)\right]
\end{aligned}
$$

In these equations, $g_{q}=2$ is the spin degeneracy in spinsaturated matter, $n_{q}^{T}$ is the finite temperature occupation number at effective chemical potential $\tilde{\mu}_{q}=\mu_{q}-\partial \mathscr{E}_{\text {pot }} / \partial n_{g q}$, $n_{q}^{T}=\left[1+\exp \left(\left(p^{2} / 2 m_{q}^{*}-\tilde{\mu}_{q}\right) / T\right)\right]^{-1}$.

The choice for the energy density $\mathscr{E}_{\text {pot }}$ and the effective nucleon mass $m_{q}^{*}$, containing the interaction effects, defines the equation of state. For the applications shown in this paper we have considered a large set of different well-known Skyrme functionals for these two quantities [30], which have been successfully confronted with different nuclear structure data and are also compatible with the most recent experimental constraints on nuclear matter properties [31-33].

In the present NSE model, the free energy density of the nucleon gas is reduced with respect to the homogeneous gas expression, Eq. (2), in order to account for the in-medium modification due to the finite volume occupied by the clusters [34, 35]. The result is:

$$
f_{g}=f_{H M}\left(1-\sum_{N, Z} n^{(N, Z)} \frac{A}{n_{0}(\boldsymbol{\delta})}\right),
$$

where the sum runs over the different clusters weighted by their multiplicity per unit volume $n^{(N, Z)} \cdot n_{0}(\delta)$ denotes the saturation density of asymmetric matter evaluated at the cluster asymmetry $\delta$, and accounts for the compressible character of the clusters. The asymmetry $\delta$ differs from the cluster global asymmetry $(N-Z) / A$ because of Coulomb and skin effects, and is additionally modified to account for the influence of the external gas [28, 36].

We have checked that, within these phenomenological limits, the choice of the interaction does not produce any sensible effect neither on the composition of matter during collapse nor on the average capture rates. This is due to the fact that, during the collapse trajectories we have considered, the density of the nucleonic gas and its neutron enrichment are not important enough to make unbound nucleon energetics play a significant role. This result is perfectly compatible with previous findings [18, 37].

The cluster multiplicities are given by the self-consistent NSE expression:

$$
\ln n^{(N, Z)}=-\frac{1}{T}\left(F_{T}-\mu_{B} A_{e}-\mu_{p} Z_{e}\right),
$$


where we have defined the bound fraction of clusters by $A_{e}=A\left(1-n_{g} / n_{0}(\delta)\right), Z_{e}=Z\left(1-n_{p g} / n_{0 p}(\delta)\right)$, with $n_{0 p}=$ $n_{0}(\delta)(1-\delta) / 2$. The chemical potentials can be expressed as a function of the gas densities only:

$$
\begin{aligned}
\mu_{B} & \equiv \frac{\partial f_{H M}}{\partial n_{g}} \\
\mu_{p} & \equiv \frac{\partial f_{H M}}{\partial n_{g p}} .
\end{aligned}
$$

In eq. (5) $F_{T}$ is the free energy of the cluster immersed in the nucleon gas:

$$
\begin{aligned}
F_{T}\left(N, Z, n_{g}, \delta_{g}\right) & =-B-T \ln \left(A_{e}^{\frac{3}{2}} c_{T} V_{\text {tot }}\right) \\
& -f_{H M}\left(n_{g}, \delta_{g}\right) \frac{A}{n_{0}(\delta)} \\
& +\delta F_{\text {Coulomb }}+\delta F_{\text {surf }},
\end{aligned}
$$

where the total volume $V_{\text {tot }}$ has been introduced. Again, because of the low gas densities considered in this application, the third and fifth term in eq. (8), representing the in-medium bulk and surface modification of the cluster free energy due to the presence of the gas, turn out to be negligible.

The most important ingredient of the NSE model is thus given by the vacuum binding energy of the cluster $B$, corrected by the well known electron screening effect $\delta F_{\text {coul }}$, and, to less extent, the cluster level density entering the temperature dependent degeneracy factor $c_{T}$ given by:

$$
c_{T}=\left(\frac{m T}{2 \pi \hbar^{2}}\right)^{3 / 2} \int_{0}^{\langle S\rangle} d E\left[D_{N, Z}(E) \exp (-E / T)\right]
$$

where $D_{N, Z}$ is the density of states of the cluster, $\langle S\rangle=$ $\min \left(\left\langle S_{n}\right\rangle,\left\langle S_{p}\right\rangle\right)$ is the average particle separation energy, $m$ is the nucleon mass. Concerning the density of states, we use a back-shifted Fermi gas model with parameters fitted from experimental data [38]. The key quantity of the model is thus the cluster binding energy, which we shall discuss in more detail in the following subsection.

\section{Mass model}

Consistency with the equation of state of the free nucleons in principle demands that the cluster mass should be evaluated with the same energy functional employed for the gas. This is indeed done in the most recent NSE models [34, 35, 39-41]. However, no functional model sufficiently precisely reproduces nuclear ground states, and when experimentally measured nuclear masses are available, they are preferentially used in all recent NSE models [28, 29, 35, 41]. It is also important to stress at this point that, independent of the nuclear matter parameters of the EoS (symmetric nuclear matter incompressibility $K_{\infty}$, symmetry energy per nucleon at the saturation density of symmetric matter $J_{0}$, slope $L$ and curvature $K_{\text {sym }}$ of symmetry energy at symmetric matter saturation density), no functional model gives completely reliable extrapolations of nuclear masses in the neutron-rich region where experimental masses are not available. This leads to a model dependence of the results, which is not related to any unknown nuclear matter parameter, but rather to the poor performance of the used Hartree-Fock or Thomas-Fermi approximations in the evaluation of nuclear masses, especially in the very neutron rich region. Very few nuclear functional models exist, with parameters fitted with the same degree of accuracy on infinite nuclear matter and to properties of finite nuclei [4246], and even in this case the behavior of nuclear mass towards the dripline is subject to great uncertainties.

Anyway, since in the collapse applications the energetics of the gas plays a negligible role, the argument of consistency is not very important. For this reason we have chosen to employ, whenever experimental measurements are missing, the Duflo-Zuker mass model [47] which gives, within a microscopically inspired formalism, the present best reproduction of measured nuclear masses. For the unbound nucleon component the SLY4 [48] Skyrme effective interaction is used.

Since we are here mainly interested in the region around the $N=50$ and $N=82$, and in order to get an idea of the performance of the different mass models, Fig. 2 shows the evolution of the two-neutron separation energy for different elements around the magic neutron numbers $N=50$ and $N=82$ as predicted by the 10 parameter model of Duflo and Zuker[47] and two other extremely successful and popular nuclear mass models, the Bruxelles functional BSK22 [49] and the Finite Range Droplet Model (FRDM) by Moller and Nix [50, 51], in comparison to experimental data from Ref. [52]. The overall dispersion of the models on the binding energy over the global mass table is $561 \mathrm{KeV}$ for DZ10, $580 \mathrm{KeV}$ for BSK22, and $656 \mathrm{KeV}$ for FRDM [49, 53] [67].

We can observe that all models appear approximately equivalent in the level of reproduction of experimental data. Their extrapolation in the neutron rich region however increasingly deviate from each other approaching the dripline. In particular, a clear and strong slope change is visible at $N=50$ and $N=82$ for all elements including the lightest ones for the phenomenological models DZ10 and FRDM. This indicates the presence of a very strong magic number, unmodified by the increasing neutron richness. Conversely the more microscopic BSK22 shows a more irregular behavior, and a certain quenching of magicity going towards the neutron dripline [54, 55], especially for $N=50$. This shell quenching appears to be closely related to the treatment of the pairing interaction. A proper inclusion of pairing correlations is certainly a very delicate issue, but one can at least expect that it should be better treated in a self-consistent Hartree-FockBogoliubov calculation such as BSK22 than in a phenomenological mass model. These differences show the difficulty of extrapolation of nuclear masses far from stability. 

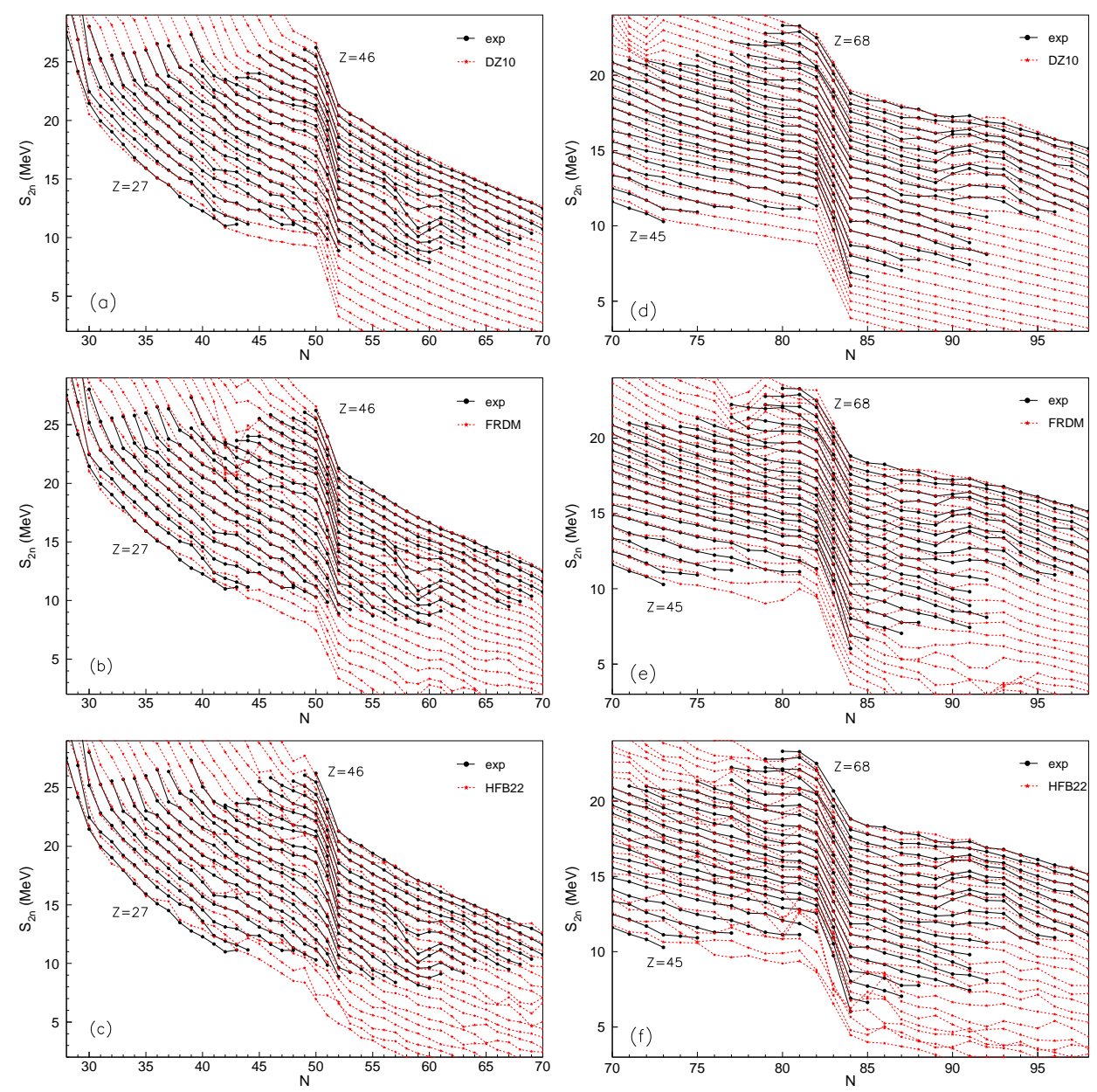

FIG. 2: (Color online) Two neutron separation energy over selected isotopic chains as predicted by different mass models (DZ10 [47], FRDM [50, 51] and HFB22 [49]) (solid stars), and in comparison to experimental (solid circles) from Ref. [52].

\section{RESULTS}

\section{A. Thermodynamic conditions during core collapse}

Exact values of temperature, baryon number density and proton fraction reached during core collapse depend on many ingredients and among others the chosen EoS and weak interaction rates. Thus, without performing a simulation, the results cannot be completely consistent. We think, however, that it is sufficient to take typical values for the purpose of the present paper, where we aim to illustrate the possible impact of a modification of nuclear masses far from stability on the electron capture rates. A detailed simulation is left for future work.

To obtain such typical values, we consider here two different core collapse trajectories from Refs. [2], as reported by Juodagalvis et al. in Ref. [25]. They correspond to the prebounce evolution for the central element of the star at an enclosed mass of 0.05 solar masses, in the case of a $15 M_{\odot}$ and a $25 M_{\odot}$ progenitor. These simulations use rates from LMSH ta- bles [21, 22] and the fully general relativistic, spherically symmetric AGILE- BOLTZTRAN code with detailed neutrino radiation hydrodynamics. For further details on the simulations, see Refs. [2]. A more complete study would certainly require a complete and consistent simulation. We expect, however, that the qualitative findings do not depend strongly on the quantitative details. In addition, it was observed [56] that the electron fraction profiles are well correlated with the density during the collapse phase, meaning that matter, independently of the exact position inside the star, will follow similar trajectories and that our example conditions taken from the central element are valid more generally.

The thermodynamic conditions during the evolution in terms of temperature, baryon number density and proton fraction are shown in Fig. 3. The vertical bar indicates the region in $Y_{p}=Y_{e}$, where the experimental information on nuclear masses starts to be incomplete according the nuclear abundances obtained within our NSE model employing experimental masses [52] complemented with the DZ10 mass table [47]. For both progenitors, this happens at densities higher than 


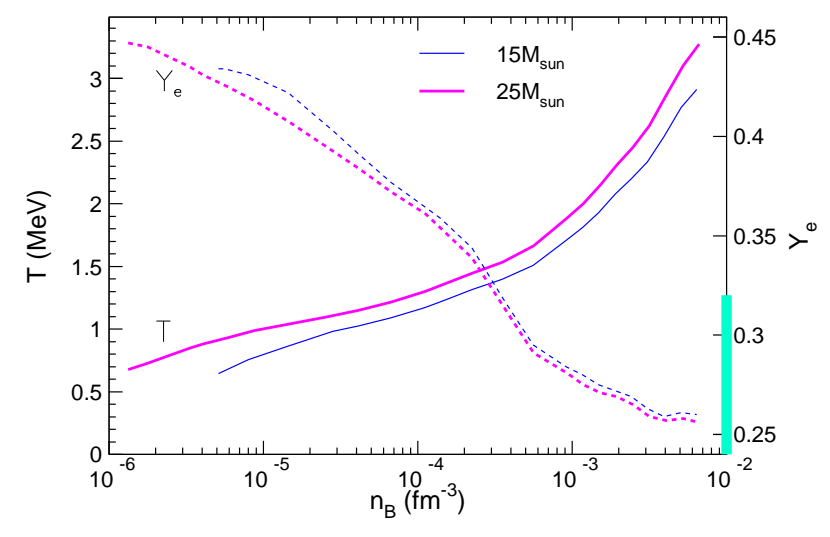

FIG. 3: (Color online) Thermodynamic conditions $\left(T, n_{B}, Y_{e}\right)$ reached by the central element in the core-collapse of two progenitor stars with zero age main sequence masses $15 M_{\odot}$ and $25 M_{\odot}$ as reported in Ref.[25]. Simulations are from Refs. [2].

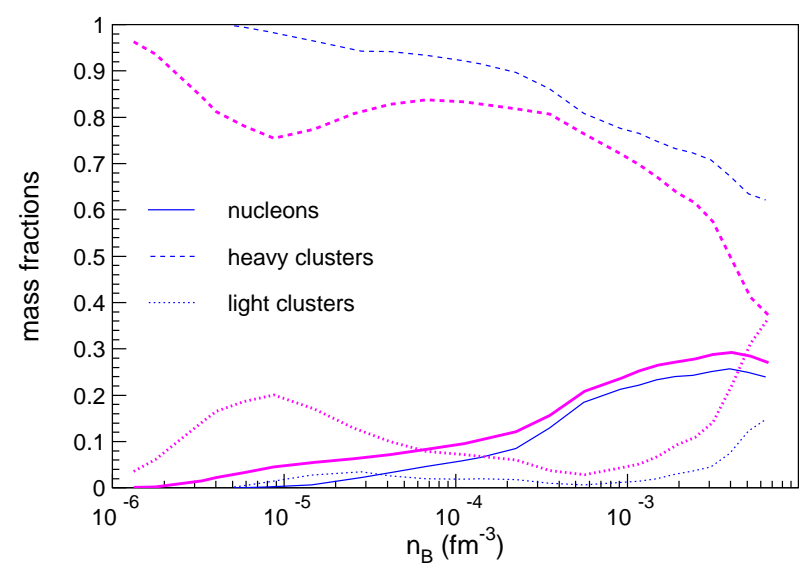

FIG. 4: (Color online) Mass fractions of free nucleons, light $(2 \leq A<20)$ and heavy $(A \geq 20)$ clusters obtained for the central element of the core-collapse with a $15 M_{\odot}$ (thin blue lines) and a $25 M_{\odot}$ (thick magenta lines) progenitor, see Ref.

[25]. The temporal evolution is labeled via the baryon number density as in Fig. 3. The color coding is the same as in Fig. 3 .

$n_{B} \approx 4 \times 10^{-4} \mathrm{fm}^{-3}$, and temperatures above $T \approx 1.4 \mathrm{MeV}$. We expect that the mass model has a considerable influence at this stage of the collapse.

\section{B. Chemical composition and capture rates}

At any time during collapse, and for both collapse trajectories, the cluster mass distribution within the NSE model, using experimental masses [52] complemented with the DZ10 mass table [47] for the nuclei for which experimental mass measurements are not available, has a multi-peaked structure. A first light cluster component exponentially decays up to a
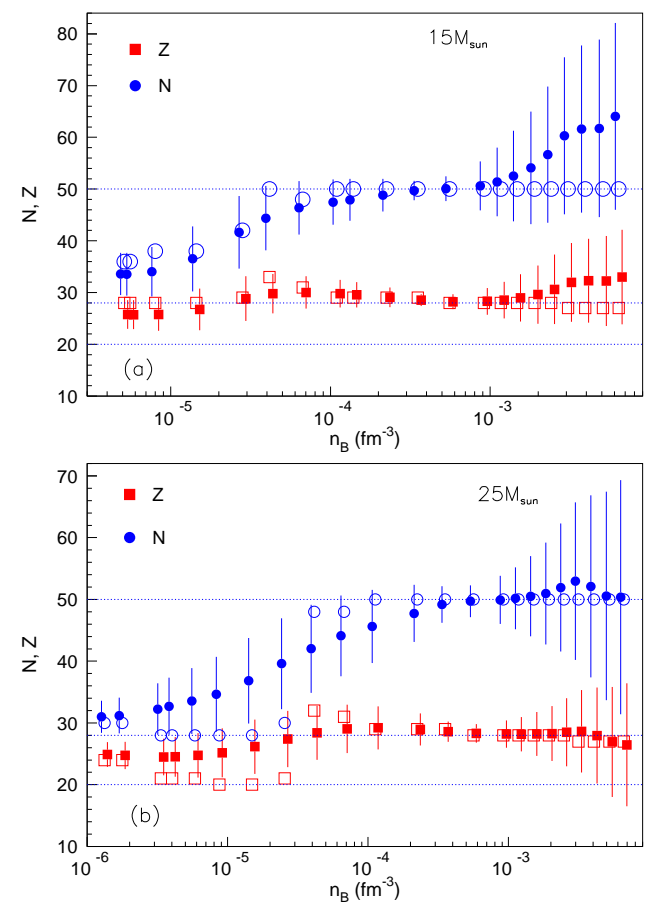

FIG. 5: (Color online) Average (solid symbols) and most probable (open symbols) proton and neutron numbers of heavy $(A \geq 20)$ nuclei produced in the central element of the core-collapsing $15 M_{\odot}$ and $25 M_{\odot}$ progenitors [6, 7, 25] as a function of baryonic density. The vertical bars correspond to the standard deviation of the distribution. For better readability, $N$ and $Z$ data have been slightly displaced in density.

minimum around $A \approx 20$; then the abundances increase and show one or several peaks in regions of the mass table which evolve according to the collapse time. To have a global glance of these distributions, Fig. 4 displays the mass fraction corresponding to nucleons, light clusters $(2 \leq A<20)$ and heavier clusters $(A \geq 20)$. The thermodynamic conditions are labeled by the baryon number density. Other mass models lead to very similar results, and we will keep DZ10 as our fiducial mass model in the subsequent calculations. We can see that the higher progenitor mass leads to a higher mass fraction for the lighter elements (nucleons and light clusters), while heavy nuclei are largely dominant at all times with the less massive progenitor at this stage of the collapse. The reason is the systematically higher temperature in the central element of the more massive star (see Fig. 31). One can observe, too, that the behavior of the clusters abundances is non-monotonic for the conditions obtained with the higher mass progenitor. This is due to the interplay between the increasing temperature during collapse, which favors light clusters with respect to heavy ones, and the increasing density leading to an opposite effect.

The composition of heavy $(A \geq 20)$ nuclei is further explored in Fig. 5] which shows for the two trajectories the average and most probable neutron and proton numbers of these nuclei. We can see that the two trajectories produce quali- 


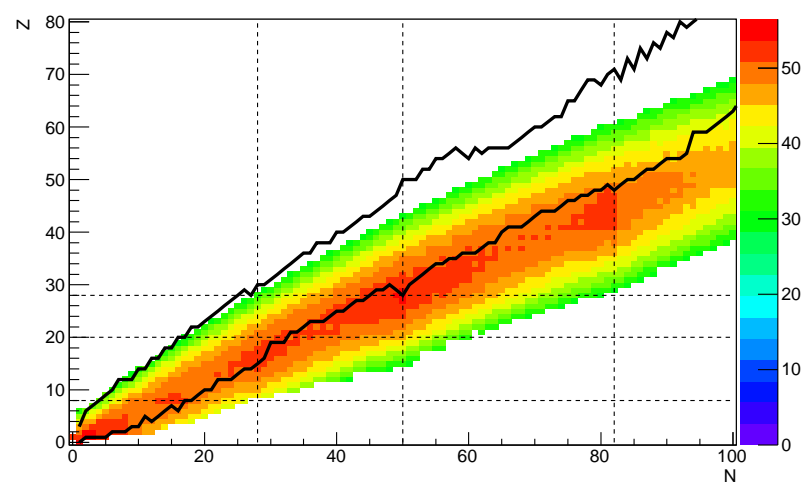

FIG. 6: (Color online) Nuclear abundances (arbitrary units) corresponding to $n_{B}=1.18 \times 10^{-3} \mathrm{fm}^{-3}, T=2 \mathrm{MeV}$, $Y_{e}=0.275$. The solid lines mark the boundaries of experimental mass measurements. The dotted lines mark magic numbers.

tatively similar patterns. More precisely, by increasing the density larger clusters are produced. Increased densities imply, along the considered trajectories, increased neutron enrichment of stellar matter which, in turn, leads to increased neutron enrichment of nuclear clusters. The standard deviation of the neutron and proton numbers distribution (signaled by the vertical bars) are never negligible meaning that treating matter composition within the Single Nucleus Approximation (SNA) would have produced erroneous results, as already acknowledged by the pioneering works [1]. In both cases and over most of the explored density range average $N$ and $Z$ numbers differ from the most probable ones. One reason is that the distributions are not only broad but multi-peaked, too. Indeed, very often the most probable nuclei are magic in $N$. Magicity in $Z$ is less frequent because of the smaller total number of protons. The reduced number of protons with respect to neutrons explains also the systematically narrower distributions in $Z$. Finally, the increasing width of the distributions in neutron number with increasing density arise due to the competition of a second magic number, in the present case $N=82$.

The stability of the fragment production pattern is in agreement with the findings by Ref. [18], who have obtained a very similar multiplicity distribution pattern for a bunch of different trajectories with different progenitor models and EoS. We therefore conclude that the present simulation is reasonably representative of the generic evolution of the central element.

To better understand the origin of this behavior, Fig 6 displays the isotopic abundances corresponding to $n_{B}=1.18 \times$ $10^{-3} \mathrm{fm}^{-3}, T=2 \mathrm{MeV}, Y_{e}=0.275$, i.e. to the time where the dispersion of the distribution of Fig. 5 starts to become nonnegligible. The distribution is centered around the $N=50$ neutron magic number, and the important width is due to the emergence of a second peak around $N=82$. This finding is in agreement with the simulations in Ref. [18] and a similar effect was observed for the neutron star crust in Refs. [57, 58]. In particular, in Ref. [18] it was shown that the overall vari-
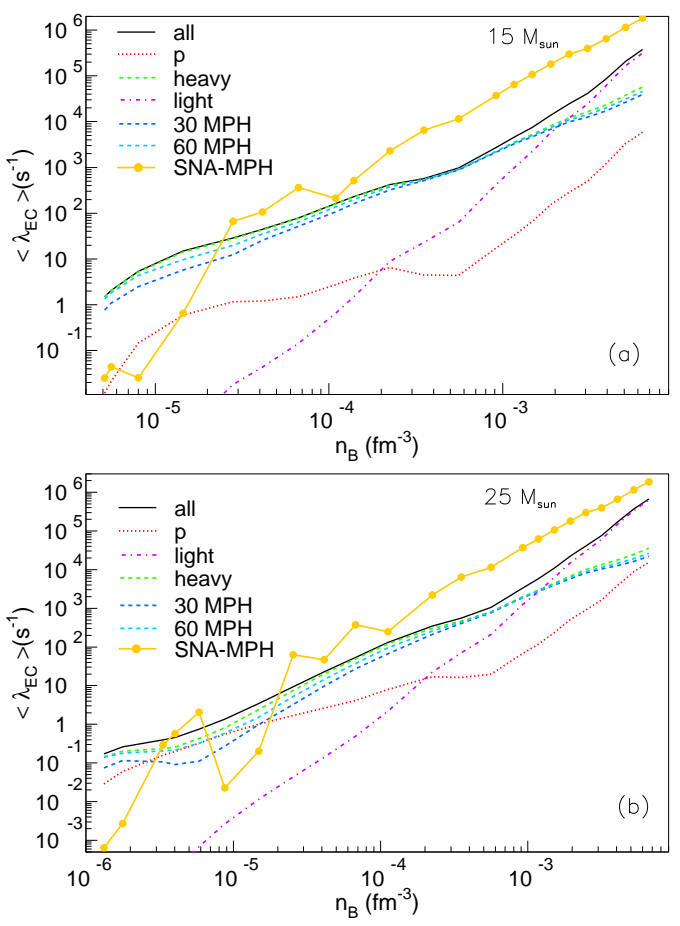

FIG. 7: (Color online) Average electron capture (EC) rates on protons, light $(2 \leq A<20)$ nuclei, heavy $(A \geq 20)$ nuclei and the 30 (dashed blue lines) and 60 (dashed cyan lines), respectively, most probable heavy nuclei. SNA-like EC rates (see text) are also plotted (SNA-MPH). The considered thermodynamical trajectories correspond to the central mass element of the $15 M_{\odot}$ and $25 M_{\odot}$-progenitors [25].

ation of the electron fraction during the collapse is most sensitive to the electron capture rate on nuclei in the mass range $74 \leq A \leq 84$, particularly on ${ }^{78} \mathrm{Ni},{ }^{79} \mathrm{Cu}$, and ${ }^{79} \mathrm{Zn}$ close to the $N=50$ shell closure. Our findings confirm these results. The two lines in Fig 6 show the borders of the region where mass measurements exist, though with a variable degree of precision. We can see that the most abundant nuclei lay just outside this border. This means that their abundance, and therefore their preeminent role in the electron capture mechanism, relies on the extrapolation of the $N=50$ and $N=82$ shell closure far from stability, in a neutron rich region where mass measurements do not exist and spectroscopic informations are scarce and incomplete. This means that small modifications in the nuclear binding energies of nuclei with masses not yet measured, but measurable in a near future, can change star matter composition and, consequently, all astrophysical quantities depending on it, notably weak rates. This point will be explored in more detail in the following subsection.

For this fiducial model, we now show the NSE averaged electron capture (EC) rates on the different species $\mathscr{C}$, $\left\langle\lambda_{E C}^{\mathscr{C}}\right\rangle=\sum_{(N, Z) \in \mathscr{C}} n^{(N, Z)} \lambda_{E C}^{(N, Z)} / \sum_{N, Z} n^{(N, Z)}$. The two above mentioned core-collapse trajectories will be considered and L03 formulae (see Sec. IIA) will be used. Let us first concentrate on the capture rates on heavy nuclei. As it is well known 
[1], a very huge number of different nuclear species contribute to the total rate in all thermodynamics conditions. This can be appreciated by limiting the rate calculation to the $N$ most probable nuclei, $\left\langle\lambda_{E C}^{N}\right\rangle=\sum_{i=1}^{N} n_{i} \lambda_{E C}^{i} / \sum_{N, Z} n^{(N, Z)}$, where $n_{i}$ is the abundance of the $i-t h$ most probable cluster. Comparing the result obtained with $N=30$ and $N=60$, with the one corresponding to the whole distribution, one can see how important it is to properly account for the complete distribution of nuclear species. In the case of both progenitors the 60 most probable nuclei never exhaust the average $\mathrm{EC}$ rate on heavy nuclei and, for instance, at $n_{B}=1.4 \times 10^{-5} \mathrm{fm}^{-3}$ they account for only $60 \%$ of $\left\langle\lambda_{E C}^{\text {heavy }}\right\rangle$.

The inadequacy of the single nucleus approximation was recently stressed in Ref.[16]. In that work it was shown that sizable differences in the collapse dynamics are obtained if the NSE model is replaced with a more conventional model [59] considering a single representative Wigner-Seitz cell for each thermodynamic condition, even if the same TM1 energy functional was employed in both models. However, in that work the individual rates were replaced by a single rate on the most probable cluster, using the simplified Bruenn parametrization [15]. Our results show that the complete nuclear distribution should also be used in the calculation of the rates. If we replace the folding of the individual rates with the EC rate of the most probable nucleus weighted by the baryon number fraction bound in clusters, $\left\langle\lambda_{E C}^{S N A}\right\rangle=\left(n_{c l} / n_{B}\right) \lambda_{E C}^{M P}$, the result (lines with points) is seen to very badly reproduce the complete folding result. As one may see in Fig. 7 at low densities $\left\langle\lambda_{E C}^{S N A}\right\rangle$ generally underestimates the NSE averaged EC rate and, because of structure effects related to the low temperatures at these densities, manifests a huge scattering. On the contrary, at higher densities and temperatures $\left(n_{B}>3 \times 10^{-5}\right.$ $\left.\mathrm{fm}^{-3}\right),\left\langle\lambda_{E C}^{S N A}\right\rangle$ largely overestimates the NSE-averaged EC rate. Obviously, different values are expected if instead of the L03 approximation, other prescriptions are employed for the individual EC rates, see the discussion in Ref. [18], too. However, the general trend induced by the different averaging procedures should remain the same.

Concerning the EC rates on light nuclei, they are negligible at the beginning of the collapse but increase strongly, mainly due to the increasing temperature, and become dominant in the latest stage. This underlines the importance of including microscopic calculation of EC rates for light nuclei, too [60].

The total rates are given by the sum of the different contributions, including electron capture on free protons (dotted lines) which however plays a minor role at all times. Comparing Figs. 3, 5] and 7, we note that for thermodynamic conditions where very neutron rich nuclei start to dominate, with masses which are not experimentally known, they still represent the major electron capture source. This qualitative picture is independent of the progenitor mass. From a more quantitative point of view, the relative importance of heavy clusters is higher for the lower mass progenitor, essentially because of the lower temperatures reached during the collapse.

\section{Evolution of magicity far from stability}

It is well known in the recent nuclear structure literature that even major shell closures can be quenched far from stability, see e.g. [61-63]. A very clear evidence exists for the $N=20$ magic number [61, 62], which corresponds to a huge gap for decreasing proton number up to ${ }^{34} \mathrm{Si}$, and suddenly disappears in the next even-even isotope ${ }^{32} \mathrm{Mg}$. This is partly due to the modification of single-particle energies far from stability, with the consequence that new magic numbers can appear corresponding to strongly deformed configurations. However the main reason of the modification of magicity relies on effects which go beyond the naive single particle shell model. First, the effect of the proton-neutron residual interaction in nuclei with strong asymmetry is decreased; moreover, correlations play an increasing role which makes the very concept of shell closure less relevant. As a consequence, the main effect is a quenching of the shell gap, even if secondary new gaps can appear in very localized regions of the nuclear chart.

The possible modification of the $N=50$ and $N=82$ shell gaps far from stability is the object of intense theoretical and experimental research in nuclear structure (e.g. [61, 63]). Here, we do not have the ambition to model this phenomenon, but simply analyze the modifications in the matter composition and associated electron capture rates, which would be induced by the expected shell quenching.

A similar idea was proposed in Ref. [64], who showed that a modified mass formula built to incorporate the possibility of shell quenching, has a striking impact on canonical calculations of the r-process. We follow a similar strategy as in Ref. [64] and introduce a modified expression for the binding energy in the following form:

$$
\begin{aligned}
B^{m}(A, Z)= & B^{\text {exp }}(A, Z), Z_{i}^{\text {exp }}(A) \leq Z \leq Z_{s}^{\text {exp }}(A), \\
= & B^{L D}(A, Z)+f\left(Z_{i}^{\text {exp }}(A)-Z, \Delta Z, \alpha\right) \\
\times & \left(B^{D Z}(A, Z)-B^{L D}(A, Z)\right) \\
& Z_{i}^{D Z}(A) \leq Z<Z_{i}^{\text {exp }}(A) \\
= & B^{L D}(A, Z)+f\left(Z-Z_{s}^{\text {exp }}(A), \Delta Z, \alpha\right) \\
\times & \left(B^{D Z}(A, Z)-B^{L D}(A, Z)\right) \\
& Z_{s}^{\exp }(A)<Z \leq Z_{s}^{D Z}(A),
\end{aligned}
$$

where $B^{\exp }(A, Z)$ and $B^{D Z}(A, Z)$ stand for the experimental binding energy [52] and, respectively, predictions of DZ10 mass model [47]. $Z_{i}^{\text {exp }}(A)$ (resp., $Z_{i}^{D Z}(A)$ ) and $Z_{s}^{\text {exp }}(A)$ (resp. $Z_{s}^{D Z}(A)$ ) correspond to the most neutron-rich and, respectively, most neutron-poor nucleus with $A$ nucleons for which experimental masses (resp., predictions of DZ10) exist. $B^{L D}(A, Z)$ is a simple liquid-drop binding energy calculated according to

$$
\begin{aligned}
B^{L D}(A, Z) & =a_{v} A-a_{s} A^{2 / 3}-a_{v i} 4 I(I+1) / A \\
& +a_{s i} 4 I(I+1) / A^{4 / 3}-a_{c} Z(Z-1) / A^{1 / 3} \\
& +V_{p}(A, Z),
\end{aligned}
$$



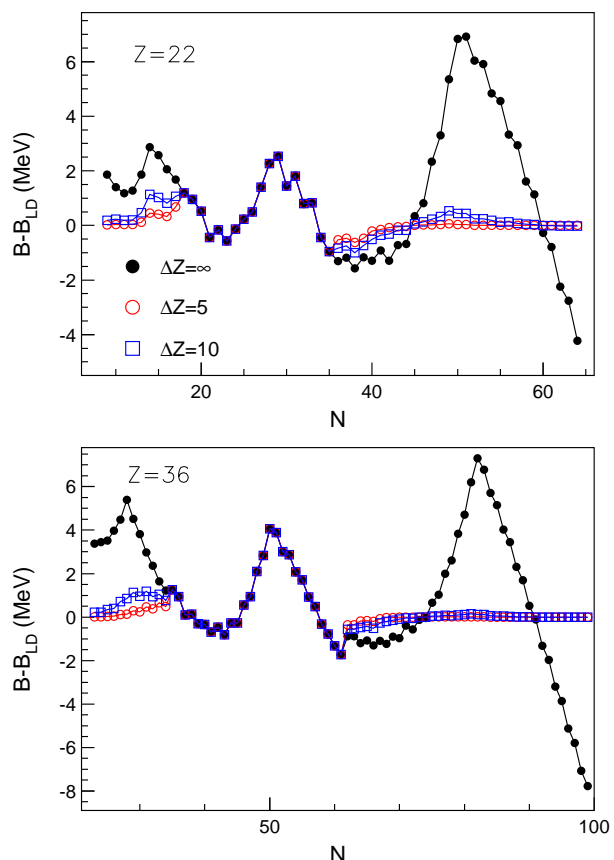

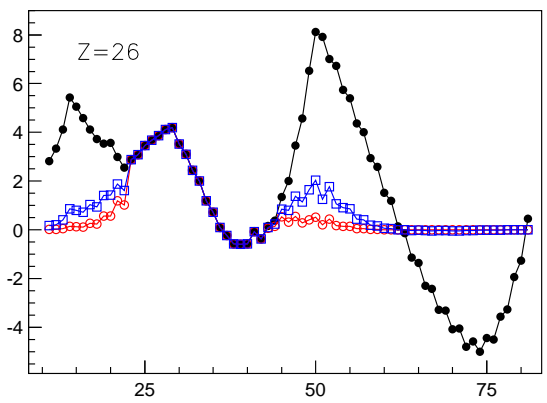

$\mathrm{N}$

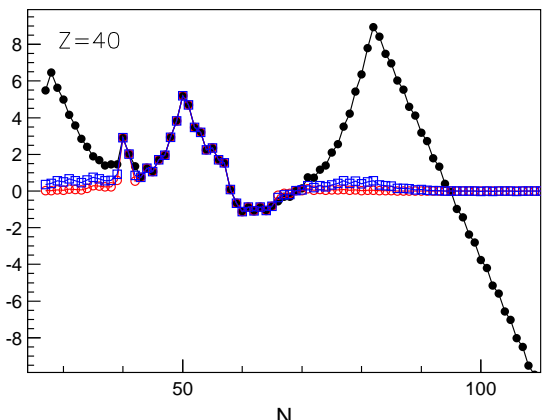

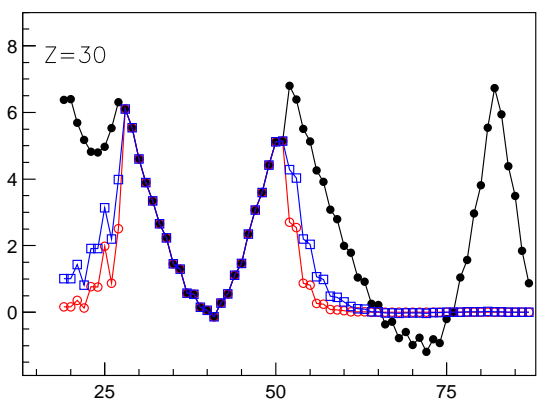

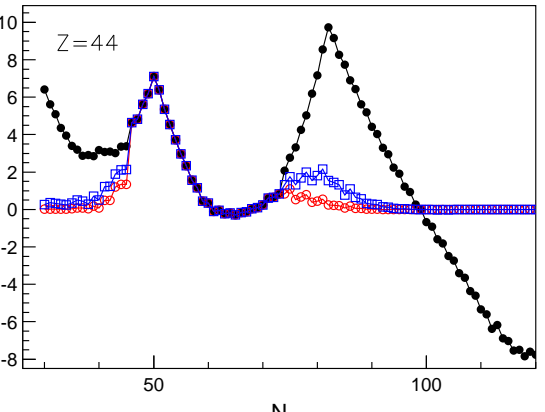

FIG. 8: (Color online) LDM-shifted binding energy as a function of neutron number for different isotopes strongly populated during core collapse. DZ10 [47] results (solid black dots) are plotted along modified results $B^{m}$ corresponding to two different scenarios of shell quenching (see text for details).

with $I=|A-2 Z| / 2, a_{v}=15.62 \mathrm{MeV}, a_{s}=17.8 \mathrm{MeV}, a_{v i}=29$ $\mathrm{MeV}, a_{s i}=38.5 \mathrm{MeV}, a_{c}=0.7 \mathrm{MeV}$ and $V_{p}= \pm 12 / \sqrt{A} \mathrm{MeV}$ for even-even (+) and, respectively, odd-odd nuclei (-).

Finally we introduce a smearing function depending on the parameters $\Delta Z$ and $\alpha<0$ which determine how sudden the shell quenching is supposed to be:

$$
f(x, \Delta Z, \alpha)=\exp [\alpha x / \Delta Z] .
$$

Small values of $\Delta Z$ correspond to maximum quenching, while in the limit $\Delta Z \rightarrow \infty$ we recover the DZ10 functional form, which predicts preserved magic numbers up to the dripline.

The behavior of the modified functional on the binding energy is illustrated in Fig. 8 for different arbitrary values of the smearing parameter $\Delta Z=5,10, \infty$ and $\alpha=\log \left(10^{-2}\right)$. A selected number of nuclei is displayed as a function of neutron number. These elements have been chosen since they are strongly populated in the later phase of the collapse.

As observed before, the DZ10 model shows an extremely pronounced shell closure at $N=50$ and $N=82$ for all elements including the exotic ones like ${ }^{72} \mathrm{Ti}$ and ${ }^{118} \mathrm{Kr}$. In the modified expression, Eq. (10), the gap is quenched far from stability, and the quenching is more or less pronounced depending on the choice of the parameter $\Delta Z$.

The effect of the modification of the mass formula on the distribution of nuclei is shown in Fig 9 for three different representative conditions during the collapse, taken from the $25 M_{\odot}$ progenitor [6, 7, 25]. Obviously, the effect of shell quenching is to reduce the size of the magic peaks and favor open shell nuclei, thus leading to a wider isotopic distribution. 


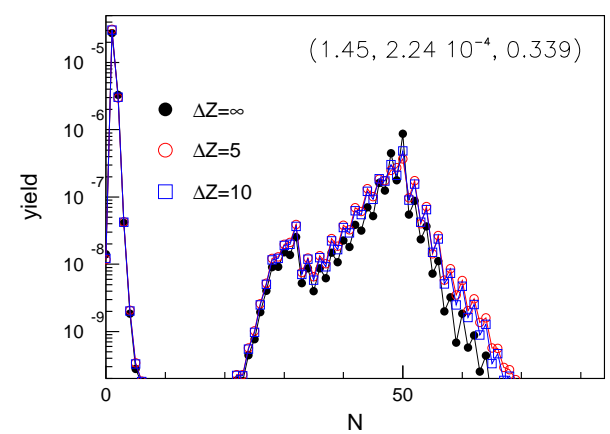

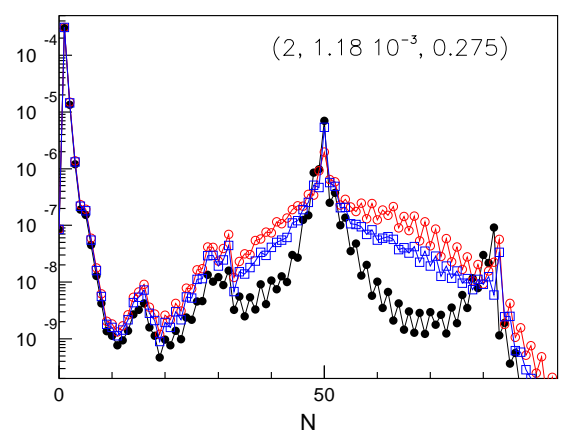

$\mathrm{N}$

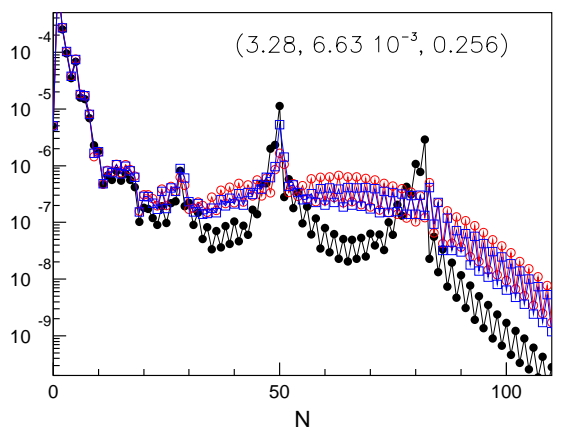

$\mathrm{N}$

FIG. 9: (Color online)

Impact of nuclear binding energy on nuclear abundances: the distribution of clusters with a given neutron number is shown. The considered thermodynamic conditions $\left(T[\mathrm{MeV}], n_{B}\left[\mathrm{fm}^{-3}\right], Y_{p}\right)$ are mentioned on each panel, corresponding to three different times in the evolution of the central element of the collapse with a $25 M_{\odot}$ progenitor [2, 25]. The same prescriptions for the binding energies as in Fig. 8 are used.

\section{Effect on the electron capture rates}

Our final result about the impact of shell quenching towards the drip-line on NSE-averaged electron capture rate is shown in Fig. 10, Several magicity quenching recipes have been considered $\Delta Z=2,5,10$ and $\alpha=\log \left(10^{-2}\right)$ for each of the two progenitors. Their predictions are plotted in terms of relative deviations with respect to the fiducial model.

Panel (a) corresponds to NSE-averaged EC rates on heavy nuclei. Independently on the stiffness of magicity quenching and progenitor mass, the same pattern is obtained. Over a certain time after neutron-rich nuclei start to be copiously populated shell quenching induces an increase of the EC rate. This is due to a significant abundance increase for non-magic nuclei and a moderate abundance decrease of the $\mathrm{N}=50$ magic nuclei. In this regime shell quenching on the $\mathrm{EC}$ rates on heavy nuclei is more pronounced, and lasts for a longer time, in the case of the $25 M_{\odot}$ progenitor. Toward the end of the considered trajectories, the opposite effect is obtained. Here shell quenching is responsible for a reduction of one order of magnitude in the abundance of $\mathrm{N}=50$ and $\mathrm{N}=82$ magic nuclei, not compensated by the abundance increase of non-magic nuclei and the corresponding increase in the $Q$-value. In the two regimes, the amplitude of the effect depends on both quenching parameter $\Delta Z$ and progenitor mass and may amount up to $30 \%$. Panel (b) shows that, despite the fact that heavy nuclei represent only a fraction of the whole mass and more isospinsymmetric light nuclei are not affected by shell quenching, modification of EC rate on heavy nuclei survives in the inclusive rate. Up to $n_{B} \lesssim 3 \times 10^{-4} \mathrm{fm}^{-3}$, where $X_{\text {heavy }} \gtrsim 0.8$ (see Fig. (4), $\left\langle\lambda_{E C}^{m}\right\rangle /\left\langle\lambda_{E C}\right\rangle \approx\left\langle\lambda_{E C}^{m}\right.$ heavy $\rangle /\left\langle\lambda_{E C}^{\text {heavy }}\right\rangle$. For the highest considered densities the overall modification of EC rates is opposite to that seen for heavy clusters. More precisely, shell quenching here points toward an increase of EC. This happens because of a more favorable production of light clusters.

\section{SUMMARY AND CONCLUSIONS}

In this paper we have examined the consequence of a possible quenching of the $N=50$ and $N=82$ shell closures on the EC rates during core collapse. As basis of the analysis, we have considered the same typical thermodynamic conditions as in Ref. [25]. They correspond to the pre-bounce evolution of the central element of the star obtained within a corecollapse simulation using two different progenitors, a $15 M_{\odot}$ and a $25 M_{\odot}$ one, from Refs. [2]. In agreement with Ref. [18] we find that the properties of very exotic nuclei around these two shell closures is a key microscopic information to predict the evolution of the electron fraction during collapse.

We have pointed out that a quenching of these shell closures compared with the popular DZ10 mass model [47] considerably affects the nuclear distribution, and consequently the EC rates during collapse. Using the standard L03 formalism for rates on individual nuclei, we have analyzed the modification of NSE average EC rates for different scenarios of shell quenching. Depending on the progenitor mass and stiffness of shell quenching modifications of EC rates of up to $30 \%$ have been obtained. We expect that such effects, once consistently included in the time dependent evolution of the collapse, have a sizable effect on neutrino emissivity and on the enclosed mass at bounce. We should mention that the L03 approximation might be questionable in this exotic region far away from the stable $f p$-shell nuclei where these formulae were adjusted. We expect, however, that more precise rates do not change our qualitative findings. The reason is that the EC rates are considerably suppressed for nuclei with a pronounced shell closure due to the energy gap in the single particle states which makes a transition more difficult.

In this work the quenching effect is governed by the parameter $\Delta Z$ whose value is chosen in an arbitrary way. More sophisticated calculations in microscopic theories, such as modern interaction configuration shell model calculations in the relevant nuclear chart region [65] are currently underway, and will give essential information on the effective importance of the shell quenching. However, the last word will be clearly 

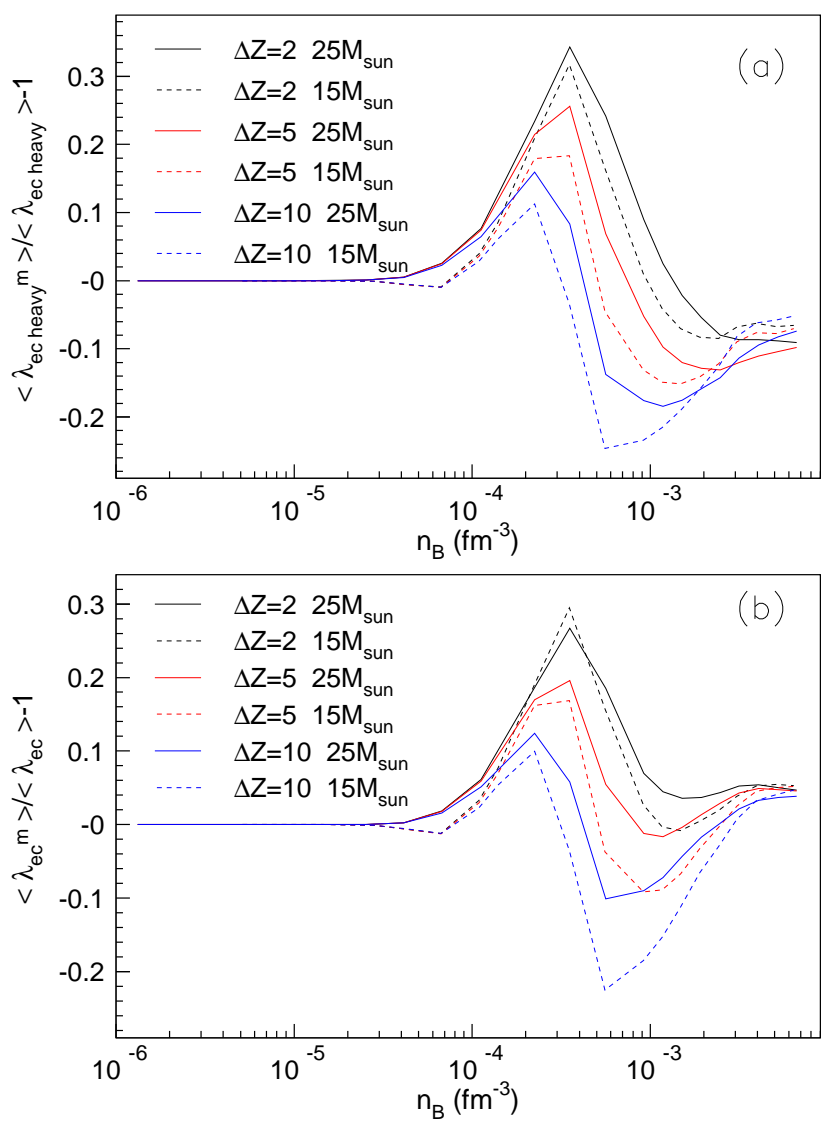

FIG. 10: (Color online) Ratio between NSE-averaged EC rates using the shell-quenched mass functional (see text) and the original DZ10 [47] mass model. The thermodynamic conditions are taken from the two core collapse trajectories of the central element of a $15 M_{\odot}$ (dashed lines) and a $25 M_{\odot}$

(full lines) progenitor [2, 25]. The temporal evolution is

labeled by the baryon number density as before. The averaged rate is calculated only on heavy nuclei $(A \geq 20)$ in the upper panel and on all nuclei in the lower panel. Different quenching factors $\Delta Z$ are considered (see text). given by phenomenology. New precise mass measurements at the edges of the known isotopic table could allow for a much better extrapolation towards the neutron rich region in a very near future.

Finally, it is important to stress that the modification of nuclear structure far from stability is expected to influence not only the nuclear mass, but also the individual EC probabilities, which should be consistently calculated together with the mass model and the energy functional describing the selfinteraction of unbound particles. Dedicated QRPA calculations are in progress to achieve this aim [66].

\section{Acknowledgments}

[1] M. B. Aufderheide, I. Fushiki, S. E. Woosley, D. A. Hartmann, Astrophys. Journ. Suppl. 91, 389 (1994).

[2] A. Heger, K. Langanke, G. Martinez-Pinedo, S.E. Woosley, Phys. Rev. Lett. 86, 1678 (2001); A. Heger, S. E. Woosley, G. Martinez-Pinedo, K. Langanke, Astrophys. J. 560, 307 (2001).

[3] G. Martinez-Pinedo, Y. H. Lam, K. Langanke, R. G. T. Zegers, C. Sullivan, Phys. Rev. C 89, 045806 (2014).

[4] K. Iwamoto, F. Brachwitz, K. Nomoto, et al. , ApJS 125, 439 (1999).

[5] F. Brachwitz et al., Astrophys. J. 536, 934 (2000).

[6] W. R. Hix, O. E. B. Messer, A. Mezzacappa, M. Liebendörfer, J. Sampaio, K. Langanke, D. J. Dean, and G. Martinez-Pinedo, Phys. Rev. Lett. 91, 201102 (2003).

[7] K. Langanke et al., Phys. Rev. Lett. 90, 241102 (2003).

[8] H. T. Janka, K. Langanke, A. Marek, G. Martinez-Pinedo and B. Mueller, Phys. Rept. 442, 38 (2007).
[9] H. Schatz, A. Aprahamian, J. Gorres, et al. Phys. Rept. 294, 167 (1998).

[10] S. Gupta, E. F. Brown, H. Schatz, P. Moeller and K. L. Kratz, Astrophys. J. 662, 1188 (2007).

[11] H. Schatz et al., Nature 505, 7481, 62 (2014).

[12] J.J. Mendoza-Temis, G. Martinez-Pinedo, K. Langanke, A. Bauswein, H. T. Janka, arXiv:1409.6135

[13] S. Goriely, A. Bauswein, O. Just, E. Pllumbi and H. T. Janka, Mon. Not. Roy. Astron. Soc. 452, 3894 (2015).

[14] H. A. Bethe, G. E. Brown, J. Applegate and J. M. Lattimer, Nucl. Phys. A324, 487 (1979).

[15] S. W. Bruenn, Astrophys. J. Suppl. 58, 771 (1985).

[16] M. Hempel, T. Fischer, J. Schaffner-Bielich, M. Liebendorfer, Astrophys. J. 748, 70 (2012).

[17] K. Langanke and G. Martnez-Pinedo, Nucl. Phys. A928, 305 (2014). 
[18] C.Sullivan, E.O'Connor, R.G.T. Zegers, T.Grubb, S.A.Austin, arXiv: 1508.07348

[19] O. Sorlin, and M.G. Porquet, Prog. Part. Nucl. Phys. 61, 602 (2008).

[20] T. Oda, M. Hino, K. Muto, M. Takahara, K. Sato, Atomic Data and Nuclear Data Tables 56, 231 (1994).

[21] K. Langanke and G. Martinez-Pinedo, Nucl. Phys. A673, 481 (2000).

[22] K. Langanke and G. Martinez-Pinedo, Atomic Data and Nuclear Data Tables 79, 1 (2001).

[23] J.-U. Nabi, H. V. Klapdor-Kleingrothaus, ADNDT 88, 237476 (2004).

[24] J. Pruet and G. M. Fuller, Astrophys. Journal Suppl. Series 149, 189 (2003).

[25] A. Juodagalvis, K. Langanke, W.R. Hix, G. Martnez-Pinedo, J.M. Sampaio, Nucl. Phys. A848, 454 (2010).

[26] G. M. Fowler, W. A. Fuller, M. J. Newman, Astrophy. J. Suppl. 48, 279 (1982).

[27] G. M. Fowler, W. A. Fuller, M. J. Newman, Astrophys. J. 293, 1 (1985).

[28] F. Gulminelli and A. R. Raduta, arXiv:1504.04493

[29] S. Burrello, M. Colonna et al., submitted to PRC.

[30] P. Danielewicz and J. Lee, Nucl. Phys. A818, 36 (2009).

[31] M. B. Tsang et al., Phys. Rev. C 86, 015803 (2012).

[32] J. M. Lattimer and Y. Lim, Astrophys. J. 771, 51 (2013).

[33] J. M. Lattimer and A. W. Steiner, Eur. Phys. J. A 50, 40 (2014).

[34] A. R. Raduta and F. Gulminelli, Phys. Rev. C 82, 065801 (2010).

[35] M. Hempel and J. Schaffner-Bielich, Nucl. Phys. A837, 210 (2010).

[36] P. Papakonstantinou, J. Margueron, F. Gulminelli and A. R. Raduta, Phys. Rev. C 88, 045805 (2013).

[37] T. Fischer, M. Hempel, I. Sagert, Y. Suwa and J. SchaffnerBielich, Eur. Phys. J. A 50, 46 (2014).

[38] T. von Egidy and D. Bucurescu, Phys. Rev. C 72, 044311 (2005); Phys. Rev. C 73, 049901 (2006).

[39] S. I. Blinnikov, I. V. Panov, M. A. Rudzsky, and K. Sumiyoshi, Astron. Astrophys. 535 A37 (2011).

[40] S. Typel, G. Röpke, T. Klähn, D. Blaschke and H. H. Wolter, Phys. Rev. C 81, 015803 (2010).

[41] S. Furusawa, S. Yamada, K. Sumiyoshi, H. Suzuki, Astrophys. J. 738, 178 (2011).

[42] L. Geng, H. Toki and J. Meng, Prog. Theor. Phys. 113, 785 (2005).

[43] S. Goriely, M. Samyn, P.-H. Heenen, J. M. Pearson and F. Tondeur, Phys. Rev. C 66, 024326 (2002).

[44] N. Chamel, A. F. Fantina, J. M. Pearson and S. Goriely, Phys.
Rev. C 84062802 (2011).

[45] J. M. Pearson, N. Chamel, A. F. Fantina and S. Goriely, Eur. Phys. J. A 50, 43 (2014).

[46] A. Y. Potekhin, A. F. Fantina, N. Chamel, J. M. Pearson and S. Goriely, Astron. Astrophys. 560, A48 (2013).

[47] J. Duflo and A. P. Zuker, Phys. Rev. C 52, R23 (1995); http://amdc.in2p3.fr/web/dz.html

[48] E. Chabanat et al., Nucl. Phys. A635, 231 (1998).

[49] S. Goriely, N. Chamel, and J. M. Pearson Phys. Rev. C 88, 024308 (2013).

[50] P. Moller and J. R. Nix, Atom. Data Nucl. Data Tabl. 26, 165 (1981).

[51] P. Moller, J. R. Nix, W. D. Myers and W. J. Swiatecki, Atom. Data Nucl. Data Tabl. 59, 185 (1995).

[52] G.Audi, M. Wang, A. H. Wapstra, F. G. Kondev, M. MacCormick, $\mathrm{X}$. $\mathrm{Xu}$, and B. Pfeiffer, Chinese Physics C36, 1287 (2012); M. Wang, G. Audi, A. H. Wapstra, F. G. Kondev, M. MacCormick, X. Xu, and B. Pfeiffer, Chinese Physics C36, 1603 (2012); http://amdc.impcas.ac.cn/evaluation/data2012/data/nubase.mas12

[53] N. Wang and M. Liu, "An improved nuclear mass formula: WS3” Journ. of Phys.: Conference Series 420, 012057 (2013).

[54] J. Dobaczewski, W. Nazarewicz and T. R. Werner, Phys. Scripta T 56, 15 (1995) .

[55] J. Dobaczewski, W. Nazarewicz, T. R. Werner, J. F. Berger, C. R. Chinn and J. Decharge, Phys. Rev. C 53, 2809 (1996).

[56] M. Liebendörfer, Astrophys. J. 633, 1042 (2005).

[57] R. N. Wolf et al. [ISOLTRAP Collaboration], Phys. Rev. Lett. 110, 041101 (2013).

[58] S. Kreim, M. Hempel, D. Lunney and J. Schaffner-Bielich, Int. J. Mass Spectr. Ion Process. 349350, 63 (2013).

[59] H. Shen, H. Toki, K. Oyamatsu, K. Sumiyoshi, Prog. Theor. Phys. 100, 1013 (1998).

[60] G. M. Fuller and C. J. Smith, Phys. Rev. D 82, 125017 (2010).

[61] O. Sorlin and M. G. Porquet, Prog. Part. Nucl. Phys. 61, 602 (2008).

[62] A. Chaudhuri et al., Phys. Rev. C 88, 054317 (2013).

[63] O. Sorlin and M.-G. Porquet, Phys. Scripta T 152, 014003 (2013).

[64] J.M. Pearson, R.C. Nayak, S. Goriely, Phys. Lett. B 387, 455 (1996)

[65] L. Coraggio, private communication.

[66] A.F. Fantina et al, in preparation.

[67] These numbers are obtained on the more restricted AME mass table from 2003. 